\title{
Anomalous diffusion under stochastic resettings: A general approach
}

\author{
Jaume Masoliver $\odot^{*}$ and Miquel Montero ${ }^{\dagger}$ \\ Department of Condensed Matter Physics and Institute of Complex Systems (UBICS), University of Barcelona, Catalonia, Spain
}

(Received 15 May 2019; published 2 October 2019)

\begin{abstract}
We present a general formulation of the resetting problem which is valid for any distribution of resetting intervals and arbitrary underlying processes. We show that in such a general case, a stationary distribution may exist even if the reset-free process is not stationary, as well as a significant decreasing in the mean first-passage time. We apply the general formalism to anomalous diffusion processes which allow simple and explicit expressions for Poissonian resetting events.
\end{abstract}

DOI: 10.1103/PhysRevE.100.042103

\section{INTRODUCTION}

There has been in recent years a great deal of work on a special type of composite random process known as stochastic resetting, which basically consists of superposition of a given underlying random process in line with the so-called resetting events that bring the process into a fixed position $x_{r}$ at random instants of time. The combined processes may have two remarkable properties since, on one hand, it may become stationary and, hence, in some way stabilized even if the underlying process is not. On the other hand, and most importantly, resettings may considerably diminish the first-passage time to any preassigned value $x_{c}$, which has a great potential of applications in many fields, especially in searching processes of all kinds, such as protein identification in DNA [1-3], animal foraging [4,5], and data mining [6-8], just to name a few. In recent years we have seen a large amount of literature on the subject of which we cite just a small sample [9-16].

To our knowledge resetting mechanisms have been amply studied when the underlying process is the Brownian motion, with some generalizations within continuous-time random walks [12], Lévy flights [13], some bounded diffusion processes $[17,18]$, and very recently to telegraphic processes [19]. Even though in most cases the resetting mechanism is governed by Poisson processes, the fact that the two key characteristics mentioned above appear for different kinds of underlying processes suggests the universal character of the resetting mechanism to stabilize the process and reduce the first-passage time, as it has been explained from diverse points of view in Refs. [14,15,20-26].

In the present paper we will insist on the universal character of the resetting mechanism by presenting a formulation of the problem for any distribution of the resetting events and underlying processes and apply the formalism for obtaining explicit expressions for anomalous diffusions under resettings. To our knowledge the study of the effect of resettings on anomalous diffusion processes has been scarcely studied.

\footnotetext{
*jaume.masoliver@ub.edu

†miquel.montero@ub.edu
}

A recent attempt in this direction is the work of Ref. [27], which is, however, limited to the spectral analysis of fractional Brownian motion with stochastic resettings. Another very recent and independent approach, which is along the lines presented here, deals with subdiffusion under resettings [28] (see also Ref. [26] for interesting asymptotic results about the same problem).

Anomalous diffusion shows up in the transport of particles through extremely disordered media (random media, fractal structures, and the like [29,30]). The most distinctive characteristic is that the mean-square displacement follows an asymptotic law of the form

$$
\left\langle x^{2}(t)\right\rangle \sim t^{\alpha}, \quad(t \rightarrow \infty, \alpha>0),
$$

showing subdiffusion when $0<\alpha<1$ and superdiffusion if $\alpha>1$. The concept of anomalous transport has been the object of very intense research during the past two decades and it extends to many areas of physical research and not only to transport phenomena (there are countless of excellent and complete reports on the subject of which we cite a very few [31-36]).

We will also address the resetting problem on anomalous diffusion processes and explore the consequences of a quite general resetting mechanism on the anomalous transport of the underlying process. There is no unique mathematical approach to anomalous diffusion processes (see Ref. [37] for a recent report focused on subdiffusive processes). Among them one of the most used approaches for anomalous diffusion is that based on the continuous-time random walk (CTRW) of Montroll and Weiss [32,38,39]. This is the procedure implicit in our development.

To this end, and in tune with Ref. [26], we will consider a broad framework in which an underlying process is brought to a fixed location at random times, drawn from an arbitrary distribution. Once we obtain general expressions for the different statistics of interest, we will specifically suppose that the reset-free process is the time-fractional Brownian motion on the line, whose probability density function (PDF) obeys the time-fractional diffusion equation:

$$
\frac{\partial^{\alpha} p}{\partial t^{\alpha}}=D \frac{\partial^{2} p}{\partial x^{2}}
$$


$(0<\alpha<2)$, where $\partial^{\alpha} / \partial t^{\alpha}$ is the fractional Caputo derivative to be formally defined in Sec. IV. Since we are interested in finding and analyzing closed formulas not only in asymptotic results [26], we will further assume that the inter-reset times are Poissonian, which is not required within our setup but approaches our results to those reported in Ref. [28] for subdiffusive processes.

The paper is organized as follows. In Sec. II we set the general formalism and obtain overall expressions for the propagator of the combined process and show the possible existence of a stationary distribution even if the reset-free process is not stationary. In Sec. III we present the general approach to the first-passage problem and obtain general expressions for the mean first-passage time showing a considerable decrease in the value of this statistic when the reset-free process has an infinite time. In Sec. IV we apply the formalism to timefractional diffusion processes and some concluding remarks are in Sec. V.

\section{GENERAL FRAMEWORK}

Let $X(t)$ be a random process on the line which, starting at $X\left(\mathcal{T}_{0}=0\right)=x_{r}$, undergoes resettings to this initial position $x_{r}$ at times $\mathcal{T}_{1}, \mathcal{T}_{2}, \ldots$, from where it continues afresh. Resettings occur at random times and we denote by $\psi_{r}(\tau)$ the PDF of the time interval between two consecutive resettings, $\tau_{n} \equiv \mathcal{T}_{n}-$ $\mathcal{T}_{n-1}, n=1,2, \ldots$,

$$
\psi_{r}(\tau) d \tau=\operatorname{Prob}\left\{\tau<\tau_{n} \leqslant \tau+d \tau\right\} .
$$

In what follows we will assume that $\psi_{r}(\tau)$ has finite moments which in particular implies a finite mean time between consecutive resettings,

$$
\left\langle\tau_{n}\right\rangle=\int_{0}^{\infty} \tau \psi_{r}(\tau) d \tau<\infty .
$$

In other words, the mean frequency of resetting (also called resetting rate) defined as

$$
r=\frac{1}{\left\langle\tau_{n}\right\rangle}>0
$$

is nonvanishing. ${ }^{1}$ The probability $\Psi_{r}(\tau)$ that no resettings occur for a time interval greater than $\tau$ is given by

$$
\Psi_{r}(\tau)=\int_{\tau}^{\infty} \psi_{r}\left(\tau^{\prime}\right) d \tau^{\prime}
$$

Before proceeding further let us note that if we keep a general resetting density $\psi_{r}(\tau)$, then the combined process with resettings is not Markovian, even if the evolution between resets is a Markov process. Specifically, this implies that the propagator $p_{r}$ of the combined process is not simply a function of the present state of the system, $X\left(t_{0}\right)=x_{0}$, but

\footnotetext{
${ }^{1}$ This assumption is needed for obtaining a stationary distribution [see Eq. (16)]. We, therefore, deal with a resetting mechanism which is not governed by a power law (see, for instance, Ref. [40] for a very recent generalization along these lines). Even so, most of the general expressions to be derived will remain valid in this case.
}

also a function of the last resetting event previous to $t_{0}$ :

$$
\begin{aligned}
& p_{r}\left(x, t \mid x_{0}, t_{0} ; x_{r}, t_{r}\right) d x \\
& \quad=\operatorname{Prob}\left\{x<X(t) \leqslant x+d x \mid X\left(t_{0}\right)=x_{0} ; X\left(t_{r}\right)=x_{r}\right\},
\end{aligned}
$$

where $t_{r}$ is the time of the last reset before $t_{0}$,

$$
t_{r} \equiv \max \left\{\mathcal{T}_{n} \mid \mathcal{T}_{n} \leqslant t_{0}\right\}
$$

The only exception to this rule is when $t_{0}$ exactly coincides with a reset, $t_{0}=t_{r}$, since then

$$
p_{r}\left(x, t \mid x_{0}, t_{0} ; x_{r}, t_{r}\right)=p_{r}\left(x, t \mid x_{0}, t_{0}\right)
$$

and, hence, $x_{0}=x_{r}$. However, for the sake of clarity in the upcoming development we will keep $x_{0}$ as the position associated to $t_{0}$, and eventually set $x_{0}=x_{r}$ when required.

To obtain an expression for the complete propagator $p_{r}$, we will first address the case when $t_{0}$ coincides with a reset time $t_{r}$ and briefly postpone the analysis of the general case. ${ }^{2}$ Note that when $t_{0}=t_{r}$ the propagator $p_{r}\left(x, t \mid x_{0}, t_{0}\right)$ obeys the following renewal equation in terms of the reset-free propagator $p_{0}\left(x, t \mid x_{0}, t_{0}\right)$ :

$$
\begin{aligned}
p_{r}\left(x, t \mid x_{0}, t_{0}\right)= & \Psi_{r}\left(t-t_{0}\right) p_{0}\left(x, t \mid x_{0}, t_{0}\right) \\
& +\int_{t_{0}}^{t} \psi_{r}\left(t^{\prime}-t_{0}\right) p_{r}\left(x, t \mid x_{r}, t^{\prime}\right) d t^{\prime},
\end{aligned}
$$

where the first term on the right-hand side accounts for the probability density when no reset event has occurred up to time $t$. The second term represents the probability density that the first resetting (ulterior to the one at $t_{0}$ and bringing again the process to $x_{r}=x_{0}$ ) occurred during any intermediate time $t_{0}<t^{\prime} \leqslant t$.

In what follows we will assume that the underlying process is not only Markovian but also time homogeneous, which implies that the propagator only depends on time differences, $p_{0}\left(x, t \mid x_{0}, t_{0}\right)=p_{0}\left(x, t-t_{0} \mid x_{0}\right)$. This leads to the conclusion that the same property holds for the combined process when $t_{0}$ coincides with a reset, that is, $p_{r}\left(x, t \mid x_{0}, t_{0}\right)=p_{r}\left(x, t-t_{0} \mid x_{0}\right)$, and Eq. (7) can be written as

$$
\begin{aligned}
p_{r}\left(x, t \mid x_{0}\right)= & \Psi_{r}(t) p_{0}\left(x, t \mid x_{0}\right) \\
& +\int_{0}^{t} \psi_{r}\left(t^{\prime}\right) p_{r}\left(x, t-t^{\prime} \mid x_{r}\right) d t^{\prime} .
\end{aligned}
$$

After taking the Laplace transform,

$$
\hat{p}_{r}\left(x, s \mid x_{0}\right)=\int_{0}^{\infty} e^{-s t} p_{r}\left(x, t \mid x_{0}\right) d t,
$$

the integral Eq. (8) reduces to the following algebraic equation:

$$
\hat{p}_{r}\left(x, s \mid x_{0}\right)=\mathcal{L}\left\{\Psi_{r}(t) p_{0}\left(x, t \mid x_{0}\right)\right\}+\hat{\psi}_{r}(s) \hat{p}_{r}\left(x, s \mid x_{r}\right),
$$

and, since $x_{0}=x_{r}$, we get [26]

$$
\hat{p}_{r}\left(x, s \mid x_{r}\right)=\frac{1}{1-\hat{\psi}_{r}(s)} \mathcal{L}\left\{\Psi_{r}(t) p_{0}\left(x, t \mid x_{r}\right)\right\} .
$$

\footnotetext{
${ }^{2}$ We will show below that in the case of Poissonian resettings for which $\psi_{r}(\tau)=r e^{-r \tau}$, as in Ref. [19], it is not necessary to consider such a distinction.
} 
In all these expressions, $\mathcal{L}\{\cdot\}$ represents the Laplace transform with respect to the time variable, and $\mathcal{L}^{-1}\{\cdot\}$ its inverse.

Taking the inverse Laplace transform of Eq. (9) we obtain the following expression for the propagator of the combined process in the form of a convolution integral:

$$
p_{r}\left(x, t \mid x_{r}\right)=\int_{0}^{t} F_{r}\left(t-t^{\prime}\right) \Psi_{r}\left(t^{\prime}\right) p_{0}\left(x, t^{\prime} \mid x_{r}\right) d t^{\prime},
$$

where

$$
F_{r}(t)=\mathcal{L}^{-1}\left\{\frac{1}{1-\hat{\psi}_{r}(s)}\right\} .
$$

Let us next consider the more general case in which $t_{0}$ does not coincide with a reset time, that is, $t_{0}>t_{r}$ and $x_{0}$ can take arbitrary values. The complete propagator, $p_{r}\left(x, t \mid x_{0}, t_{0} ; x_{r}, t_{r}\right)$, is now given by

$$
\begin{aligned}
p_{r}\left(x, t \mid x_{0}, t_{0} ; x_{r}, t_{r}\right)= & \frac{\Psi_{r}\left(t-t_{r}\right)}{\Psi_{r}\left(t_{0}-t_{r}\right)} p_{0}\left(x, t-t_{0} \mid x_{0}\right) \\
& +\int_{t_{0}}^{t} \frac{\psi_{r}\left(t^{\prime}-t_{r}\right)}{\Psi_{r}\left(t_{0}-t_{r}\right)} p_{r}\left(x, t-t^{\prime} \mid x_{r}\right) d t^{\prime},
\end{aligned}
$$

where $p_{r}\left(x, t-t^{\prime} \mid x_{r}\right)$ is given by Eq. (10). Equation (12) is the generalization of Eq. (7) in which the probability of having a reset at time $t$ after $t_{0}=t_{r}$ has been replaced by the conditional probability of having a reset at time $t$ knowing that no reset has occurred yet at $t_{0}\left(t_{r}<t_{0} \leqslant t\right)$, that is,

$$
\psi_{r}\left(t-t_{0}\right) \longrightarrow \frac{\psi_{r}\left(t-t_{r}\right)}{\int_{t_{0}}^{\infty} \psi_{r}\left(t-t_{r}\right) d t}=\frac{\psi_{r}\left(t-t_{r}\right)}{\Psi_{r}\left(t_{0}-t_{r}\right)} .
$$

Note also that in general $p_{r}\left(x, t \mid x_{0}, t_{0} ; x_{r}, t_{r}\right)$ is a function of two time intervals $t-t_{r}$ and $t_{0}-t_{r}$, except for Poissonian reset times. Indeed, if the random instants of time when resettings occur are a Poissonian set of events, then the density $\psi_{r}(\tau)$ and the probability $\Psi_{r}(\tau)$ are given by

$$
\psi_{r}(\tau)=r e^{-r \tau}, \quad \Psi_{r}(\tau)=e^{-r \tau},
$$

where $r$ is the mean rate of resettings. In such a case,

$$
\frac{\psi_{r}\left(t-t_{r}\right)}{\Psi_{r}\left(t_{0}-t_{r}\right)}=r e^{-r\left(t-t_{0}\right)}, \quad \frac{\Psi_{r}\left(t-t_{r}\right)}{\Psi_{r}\left(t_{0}-t_{r}\right)}=e^{-r\left(t-t_{0}\right)},
$$

and from Eq. (12) we see that $p_{r}\left(x, t \mid x_{0}, t_{0} ; x_{r}, t_{r}\right)$ is no longer a function of $t_{r}$, and the process becomes Markovian. ${ }^{3}$

\section{The stationary distribution}

We know that the stationary distribution is defined as the long-time limit of the propagator:

$$
p_{r}^{(\mathrm{st})}(x) \equiv \lim _{t \rightarrow \infty} p_{r}\left(x, t \mid x_{0}, t_{0} ; x_{r}, t_{r}\right) .
$$

From Eq. (12) and recalling the definition of $\Psi_{r}(t)$ we have

$$
p_{r}^{(\mathrm{st})}(x)=\lim _{t \rightarrow \infty} p_{r}\left(x, t \mid x_{r}\right)
$$

\footnotetext{
${ }^{3}$ Obviously, $p_{r}\left(x, t \mid x_{0}, t_{0}\right)$ still depends on $x_{r}$.
}

which, using a well-known property of the Laplace transform, can be written as

$$
p_{r}^{(\mathrm{st})}(x)=\lim _{s \rightarrow 0}\left[s \hat{p}_{r}\left(x, s \mid x_{r}\right)\right],
$$

and from Eq. (9) we conclude that

$$
p_{r}^{(\mathrm{st})}(x)=\lim _{s \rightarrow 0}\left[\frac{s}{1-\hat{\psi}_{r}(s)} \int_{0}^{\infty} e^{-s t} \Psi_{r}(t) p_{0}\left(x, t \mid x_{r}\right) d t\right] .
$$

Note that we are dealing with a time-interval PDF $\psi_{r}(t)$ having finite moments which implies that the Laplace transform $\hat{\psi}_{r}(s)$ can be expanded as

$$
\hat{\psi}_{r}(s)=1-\frac{s}{r}+O\left(s^{2}\right),
$$

where $r=\left\langle\tau_{n}\right\rangle^{-1}$ is the mean rate of resettings. Substituting into Eq. (15) yields

$$
p_{r}^{(\mathrm{st})}(x)=r \int_{0}^{\infty} \Psi_{r}(t) p_{0}\left(x, t \mid x_{r}\right) d t .
$$

Let us recall that a random process is stationary if there exist a nonnull stationary density $p_{r}^{(\text {st) }}(x)$ defined as the longtime limit of the complete propagator $p_{r}\left(x, t \mid x_{0}, t_{0} ; x_{r}, t_{r}\right)$, which is independent of $x_{0}$. Note that if $p_{r}^{\text {(st) }}(x)=0$ the process in the long-time limit cannot be found at some state $x$. In other words, the existence of $p_{r}^{(\text {st) }}(x) \neq 0$ stabilizes the process around some equilibrium point which in our case is the resetting position $x_{r}$.

In the present case of a combined process with stochastic resettings, the stationary distribution in the most general case is given by Eq. (16). Let us note that, attending the positive character of both $\Psi_{r}(t)$ and $p_{0}\left(x, t \mid x_{0}\right)$, the integral in Eq. (16) will not vanish in general, even if

$$
p_{0}^{(\mathrm{st})}(x)=\lim _{t \rightarrow \infty} p_{0}\left(x, t \mid x_{0}\right)=0 .
$$

That is to say, the combined process is always stationary regardless the stationary character of the underlying, resetfree, process. This proves, in a rather general manner, the stabilizing effect of resettings which otherwise is a rather intuitive effect.

For Poissonian resettings the expression given by Eq. (16) is simpler and more explicit. In this case, $\Psi_{r}(t)=e^{-r t}$ and Eq. (16) reduces to

$$
p_{r}^{(\mathrm{st})}(x)=r \hat{p}_{0}\left(x, r \mid x_{r}\right)
$$

where $\hat{p}_{0}\left(x, s \mid x_{r}\right)$ is the Laplace transform of the reset-free propagator. In this case the possible stationarity of the combined process depends on the existence of the Laplace transform of the reset-free propagator.

\section{THE FIRST-PASSAGE PROBLEM}

We next address within the general framework described above the first-passage problem for a random process with stochastic resettings. This has been the object of intense research because resettings may significantly reduce the meanfirst passage time, a fact with many practical applications and that, in particular, optimizes any search process based on the combined process. 
The characterization of the first-passage problem under resettings has been mostly addressed for the Brownian motion (i.e., unbounded diffusion process) under Poissonian resettings even though there have been recent works stressing the universal character of resetting processes [14,15,24,25]. In this section we present another view on this universal character.

Let us focus on the first-passage problem to some preassigned value $x_{c}$, also called critical value or threshold. The problem is characterized by the survival probability (SP) to threshold $x_{c}, S_{r}\left(t \mid x_{0}, t_{0} ; x_{r}, t_{r}\right)$, which is the probability that the process, being in $x_{0}$ at $t_{0}$, does not reach $x_{c}$ during the interval $\left[t_{0}, t\right]$. As before $t_{r}$ denotes the last reset time prior to $t_{0}, t_{r} \leqslant$ $t_{0}$. For diffusion processes with Poissonian resettings [9] the survival probability obeys an inhomogeneous Fokker-Planck equation with initial and boundary conditions, respectively, given by $S_{r}\left(t_{0} \mid x_{0}, t_{0}\right)=1$ and $S_{r}\left(t \mid x_{c}, t_{0}\right)=0$, independent of $t_{r}{ }^{4}$

As in the previous section with the analysis of the propagator and the stationary distribution, we can also obtain a general equation for the survival probability for any distribution of resetting events and any class of underlying process. Such an equation is an integral equation based on the renewal principle and a version of it has been recently used by Pal, Kundu, and Evans [11] in their study of diffusions with time-dependent resetting rates.

Let us denote by $S_{0}\left(t \mid x_{0}, t_{0}\right)$ the survival probability to threshold $x_{c}$ for the underlying (i.e., reset-free) process, while $S_{r}\left(t \mid x_{0}, t_{0} ; x_{r}, t_{r}\right)$ denotes the SP to $x_{c}$ for the combined process with resettings. Once again, we begin by assuming that at time $t_{0}$ a resetting event has occurred which implies that $t_{r}=t_{0}$ and $x_{r}=x_{0}$. In this case, the integral equation for the $\mathrm{SP}$ of the combined process $S_{r}\left(t \mid x_{0}, t_{0}\right)$ is

$$
\begin{aligned}
S_{r}\left(t \mid x_{0}, t_{0}\right)= & \Psi_{r}\left(t-t_{0}\right) S_{0}\left(t \mid x_{0}, t_{0}\right) \\
& +\int_{t_{0}}^{t} \psi_{r}\left(t^{\prime}-t_{0}\right) S_{0}\left(t^{\prime} \mid x_{0}, t_{0}\right) S_{r}\left(t \mid x_{r}, t^{\prime}\right) d t^{\prime},
\end{aligned}
$$

where the first term on the right-hand side gives the probability that neither a reset has occurred at time $t$ nor any hitting to threshold $x_{c}$ between $t_{0}$ and $t$. The second term represents the probability that the first resetting (after the one at $t_{0}$ ) to position $x_{r}{ }^{5}$ occurred at some instant of time $t^{\prime}$ with no hitting to $x_{c}$ between $t_{0}$ and $t^{\prime}$ and no hitting either from $t^{\prime}$ and $t$, all of this integrated over any intermediate time $t^{\prime} \in\left[t_{0}, t\right]$.

Since we are assuming time homogeneity we have that $S_{0}\left(t \mid x_{0}, t_{0}\right)=S_{0}\left(t-t_{0} \mid x_{0}\right)$ and similarly for $S_{r}\left(t \mid x_{0}, t_{0}\right)$. This allows us to set $t_{0}=0$ and rewrite Eq. (18) in the simpler form:

$$
\begin{aligned}
S_{r}\left(t \mid x_{0}\right)= & \Psi_{r}(t) S_{0}\left(t \mid x_{0}\right) \\
& +\int_{0}^{t} \psi_{r}\left(t^{\prime}\right) S_{0}\left(t^{\prime} \mid x_{0}\right) S_{r}\left(t-t^{\prime} \mid x_{r}\right) d t^{\prime} .
\end{aligned}
$$

\footnotetext{
${ }^{4}$ For telegraphic processes with Poissonian resettings $S_{r}\left(t \mid x_{0}, t_{0}\right)$ obeys an inhomogeneous telegrapher's equation [19].

${ }^{5}$ In fact $x_{r}=x_{0}$ although we keep $x_{0}$ and $x_{r}$ for the clearness of the exposition.
}

We can easily solve this integral equation in the Laplace space. To this end we first define the auxiliary quantities

$$
H\left(t \mid x_{0}\right) \equiv \Psi_{r}(t) S_{0}\left(t \mid x_{0}\right), \quad h\left(t \mid x_{0}\right) \equiv \psi_{r}(t) S_{0}\left(t \mid x_{0}\right),
$$

which allow us to write Eq. (19) as

$$
S_{r}\left(t \mid x_{0}\right)=H\left(t \mid x_{0}\right)+\int_{0}^{t} h\left(t^{\prime} \mid x_{0}\right) S_{r}\left(t-t^{\prime} \mid x_{r}\right) d t^{\prime} .
$$

The Laplace transform,

$$
\hat{S}_{r}\left(s \mid x_{0}\right)=\int_{0}^{\infty} e^{-s t} S_{r}\left(t \mid x_{0}\right) d t,
$$

turns Eq. (21) into the simple algebraic equation

$$
\hat{S}_{r}\left(s \mid x_{0}\right)=\hat{H}\left(s \mid x_{0}\right)+\hat{h}\left(s \mid x_{0}\right) \hat{S}_{r}\left(s \mid x_{r}\right),
$$

whose solution reads (recall that here $x_{0}=x_{r}$ )

$$
\hat{S}_{r}\left(s \mid x_{r}\right)=\frac{\hat{H}\left(s \mid x_{r}\right)}{1-\hat{h}\left(s \mid x_{r}\right)},
$$

where $\hat{H}\left(s \mid x_{r}\right)$ and $\hat{h}\left(s \mid x_{r}\right)$ are the Laplace transform of the functions $H\left(t \mid x_{r}\right)$ and $h\left(t \mid x_{r}\right)$ defined in Eq. (20).

As in the previous section, we can recover the general solution for the SP $S_{r}\left(t \mid x_{0}, t_{0} ; x_{r}, t_{r}\right)$, when $t_{r} \leqslant t_{0}$ and $x_{0}$ is any point, by using the rule Eq. (13) in Eq. (18):

$$
\begin{aligned}
S_{r}\left(t \mid x_{0}, t_{0} ; x_{r}, t_{r}\right)= & \frac{\Psi_{r}\left(t-t_{r}\right)}{\Psi_{r}\left(t_{0}-t_{r}\right)} S_{0}\left(t-t_{0} \mid x_{0}\right) \\
& +\int_{t_{0}}^{t} \frac{\psi_{r}\left(t^{\prime}-t_{r}\right)}{\Psi_{r}\left(t_{0}-t_{r}\right)} S_{0}\left(t^{\prime}-t_{0} \mid x_{0}\right) \\
& \times S_{r}\left(t-t^{\prime} \mid x_{r}\right) d t^{\prime},
\end{aligned}
$$

where $S_{r}\left(t-t^{\prime} \mid x_{r}\right)$ appearing on the right-hand side is given by Laplace inverting Eq. (23). Let us, however, note that $S_{r}\left(t \mid x_{0}, t_{0} ; x_{r}, t_{r}\right)$ thus defined does not guarantee that the process has never reached $x_{c}$ before $t_{0}$ and, in particular, during the interval $\left[t_{r}, t_{0}\right)$. Indeed, recall that $t_{r}$ is the time of the last reset before $t_{0}$, in consequence the evolution of the process between $t_{r}$ and $t_{0}$ could have perfectly crossed threshold $x_{c}$ and go afterwards to $x_{0}$ at time $t_{0}>t_{r}$. To avoid that eventuality we must perform the additional replacement

$$
\begin{aligned}
S_{0}\left(t-t_{0} \mid x_{0}\right) \longrightarrow & S_{0}\left(t-t_{0} \mid x_{0}\right) \\
& \times\left[1-\frac{p_{0}\left(2 x_{c}-x_{0}, t_{0}-t_{r} \mid x_{r}\right)}{p_{0}\left(x_{0}, t_{0}-t_{r} \mid x_{r}\right)}\right]
\end{aligned}
$$

in Eq. (24), where we have applied the reflection principle which is valid for underlying processes that are symmetric and space homogeneous [41].

In what follows we will, therefore, restrict our attention to the situation in which $t_{0}=t_{r}$ and thus $x_{0}=x_{r}$. In such a case and taking into account time homogeneity we can set $t_{0}=0$ and the SP is given by the Laplace inversion of Eq. (23). Knowing $S_{r}\left(t \mid x_{0}\right)$, the mean first-passage time (MFPT) to threshold $x_{c}$ from $x_{0}$ in the presence of random resettings is given by the integral

$$
T_{r}\left(x_{0}\right)=\int_{0}^{\infty} S_{r}\left(t \mid x_{0}\right) d t
$$


which in terms of the Laplace transform of the SP is simply given by

$$
T_{r}\left(x_{0}\right)=\hat{S}_{r}\left(s=0 \mid x_{0}\right) .
$$

From Eqs. (20), (23), and (25) we see that

$$
T_{r}\left(x_{0}\right)=\frac{\int_{0}^{\infty} \Psi_{r}(t) S_{0}\left(t \mid x_{0}\right) d t}{1-\int_{0}^{\infty} \psi_{r}(t) S_{0}\left(t \mid x_{0}\right) d t} .
$$

Therefore, the MFPT will be finite as long as the integrals in the right-hand side of Eq. (26) exist even if the MFPT for the reset-free process,

$$
T_{0}\left(x_{0}\right)=\int_{0}^{\infty} S_{0}\left(t \mid x_{0}\right) d t
$$

is infinite. Since the existence of the integrals in Eq. (26) is ensured for a wide class of sojourn densities $\psi_{r}(t)$ that have finite moments, we see that resettings may considerably reduce the MFPT with the subsequent increase of efficiency.

A key property of Poissonian resettings on diffusion processes is the nonmonotonous behavior of the MFPT as a function of the resetting rate $r$. In other words, the MFPT attains a minimum for a particular value of $r$. Let us now argue that this characteristic is kept for a wide class of resetting mechanisms and underlying processes. Indeed, as seen in Sec. II, any resetting mechanism governed by a PDF $\psi_{r}(t)$ with finite and nonzero mean, has a finite resetting rate $r$ defined in Eq. (4). Note that as $r \rightarrow 0$ the complete process approaches the reset-free process, so that $T_{r}\left(x_{0}\right) \rightarrow T_{0}\left(x_{0}\right)$. Let us also assume that, as in Brownian motion, $T_{0}\left(x_{0}\right)=\infty$. Therefore,

$$
\lim _{r \rightarrow 0} T_{r}\left(x_{0}\right)=\infty .
$$

However, as $r \rightarrow \infty$ the average time between consecutive resettings tends to 0 which means that the system becomes circumscribed to a shrinking neighborhood around $x_{0}=x_{r}$ if the underlying process is continuous enough. Let us further assume that $r$ is the only scale parameter of $\psi_{r}(t)$, that is,

$$
\psi_{r}(t)=r f(r t)
$$

for a certain nonnegative function $f(u)$ which, due to the normalization condition of $\psi_{r}(t)$ and Eqs. (3) and (4), satisfies

$$
\int_{0}^{\infty} f(u) d u=\int_{0}^{\infty} u f(u) d u=1 .
$$

Therefore, the scaling Eq. (28) allows us to express Eq. (26) as

$$
T_{r}\left(x_{0}\right)=\frac{\int_{0}^{\infty} d u f(u) \int_{0}^{u / r} S_{0}\left(t \mid x_{0}\right) d t}{1-\int_{0}^{\infty} f(u) S_{0}\left(u / r \mid x_{0}\right) d t} .
$$

Taking the limit $r \rightarrow \infty$, applying L'Hôpital's rule and bearing in mind the initial condition $S_{0}\left(0 \mid x_{0}\right)=1$ and Eq. (29), we get

$$
\lim _{r \rightarrow \infty} T_{r}\left(x_{0}\right)=-\frac{1}{\left.\partial_{t} S_{0}\left(t \mid x_{0}\right)\right|_{t=0}},
$$

whenever $x_{c} \neq x_{0}$. Hence, as long as $S_{0}\left(t \mid x_{0}\right)$ satisfies the condition

$$
\left.\partial_{t} S_{0}\left(t \mid x_{0}\right)\right|_{t=0}=0,
$$

the MFPT also becomes infinite as $r \rightarrow \infty$ :

$$
\lim _{r \rightarrow \infty} T_{r}\left(x_{0}\right)=\infty .
$$

Since under mild conditions $T_{r}\left(x_{0}\right)$ is a continuous function of $r$, from Eqs. (27) and (33) we see that the MFPT must attain a minimum value for $r$ somewhere in between 0 and $\infty$. This is a complementary discussion to that of Refs. $[14,15,20-25]$ on the generality of resettings.

For Poissonian resettings, the above expressions are simpler and more explicit. Indeed, substituting the exponential forms given in Eq. (14) into the definitions Eq. (20) of the auxiliary quantities $h\left(t \mid x_{0}\right)$ and $H\left(t \mid x_{0}\right)$ and taking the Laplace transform we get

$$
\hat{H}\left(s \mid x_{0}\right)=\hat{S}_{0}\left(r+s \mid x_{0}\right), \quad \hat{h}\left(s \mid x_{0}\right)=r \hat{S}_{0}\left(r+s \mid x_{0}\right),
$$

which finally results in [9]

$$
\hat{S}_{r}\left(s \mid x_{0}\right)=\frac{\hat{S}_{0}\left(r+s \mid x_{0}\right)}{1-r \hat{S}_{0}\left(r+s \mid x_{0}\right)}
$$

and

$$
T_{r}\left(x_{0}\right)=\frac{\hat{S}_{0}\left(r \mid x_{0}\right)}{1-r \hat{S}_{0}\left(r \mid x_{0}\right)} .
$$

This last equation showing that for Poissonian resettings the MFPT of the combined process is finite regardless the exact nature of the reset-free process which is a direct consequence of the fact that the Laplace transform of any survival probability always exists. ${ }^{6}$

\section{ANOMALOUS DIFFUSION AND RESETTINGS}

We will next address the resetting problem when the underlying is a time-fractional diffusive process or fractional Brownian motion.

\section{A. Stationary distribution}

In this case the propagator of the reset-free process, $p_{0}\left(x, t \mid x_{0}\right)$, obeys the time-fractional diffusion-wave equation $[19,39,42]$

$$
\frac{\partial^{\alpha} p_{0}\left(x, t \mid x_{0}\right)}{\partial t^{\alpha}}=D \frac{\partial^{2} p_{0}\left(x, t \mid x_{0}\right)}{\partial x^{2}},
$$

$(0<\alpha<2)^{7}$ with the initial condition

$$
p_{0}\left(x, t=0 \mid x_{0}\right)=\delta\left(x-x_{0}\right) .
$$

\footnotetext{
${ }^{6}$ Indeed, the survival probability (like any probability) is always less or equal to one, $S_{0}\left(t \mid x_{0}\right) \leqslant 1$, and hence $\hat{S}_{0}\left(s \mid x_{0}\right) \leqslant 1 / s$ is finite (we thank an anonymous referee for recalling us this elementary result).

${ }^{7}$ In the literature the most frequently studied case of time-fractional diffusion equation corresponds to the subdiffusive case where $0<$ $\alpha<1$. In our opinion the main reason for such a restriction on the values $\alpha$ can take lies in the fact that this subdiffusive case can be easily derived from microscopic models based on the continuous time random walk (see, for instance, Ref. [44] for a short review). However, the superdiffusive case where $1<\alpha<2$, which is also included in the time-fractional diffusion-wave Eq. (36), can be derived from microscopic models based on the fractional persistent random walk (see Ref. [39] for details).
} 
When $1<\alpha<2$ the initial condition Eq. (37) has to be supplemented with a second initial condition, which usually is

$$
\left.\frac{\partial p_{0}\left(x, t \mid x_{0}\right)}{\partial t}\right|_{t=0}=0 .
$$

The operator $\partial^{\alpha} / \partial t^{\alpha}$ is the fractional Caputo derivative defined as

$$
\frac{\partial^{\alpha} \phi(t)}{\partial t^{\alpha}}= \begin{cases}\frac{1}{\Gamma(n-\alpha)} \int_{0}^{t} \frac{\phi^{(n)}\left(t^{\prime}\right) d t^{\prime}}{\left(t-t^{\prime}\right)^{1+\alpha-n}}, & n-1<\alpha<n, \\ \phi^{(n)}(t), & \alpha=n\end{cases}
$$

$(n=1,2,3, \ldots)$. Using this definition the Laplace transform of the Caputo derivative is found to be $[43,44]$

$$
\mathcal{L}\left\{\frac{\partial^{\alpha} \phi(t)}{\partial t^{\alpha}}\right\}=s^{\alpha} \hat{\phi}(s)-s^{\alpha-1} \phi(0)-\sum_{j=1}^{n-1} s^{\alpha-1-j} \phi^{(j)}(0)
$$

$(n=1,2,3, \ldots ; n-1<\alpha<n), \quad$ where $\hat{\phi}(s)=\mathcal{L}\{\phi(t)\}$. Note that when $0<\alpha<1$ we have $n=1$ and this transform reduces to

$$
\mathcal{L}\left\{\frac{\partial^{\alpha} \phi(t)}{\partial t^{\alpha}}\right\}=s^{\alpha} \hat{\phi}(s)-s^{\alpha-1} \phi(0) .
$$

We also observe that if $1<\alpha<2$ but $\phi^{\prime}(0)=0$, the Laplace transform for the Caputo derivative is also given by Eq. (41).

The joint Fourier-Laplace transform of the free propagator $p_{0}\left(x, t \mid x_{0}\right)$ is defined as

$$
\hat{\tilde{p}_{0}}\left(\omega, s \mid x_{0}\right)=\int_{-\infty}^{\infty} e^{-i \omega x} d x \int_{0}^{\infty} e^{-s t} p_{0}\left(x, t \mid x_{0}\right) d t,
$$

and the use of Eq. (41) leads to the following solution of the initial-value problem Eqs. (36)-(38),

$$
\hat{\tilde{p}}_{0}\left(\omega, s \mid x_{0}\right)=\frac{s^{\alpha-1} e^{-i \omega x_{0}}}{s^{\alpha}+D \omega^{2}} .
$$

Recalling the Fourier inversion formula

$$
\mathcal{F}^{-1}\left\{\frac{a e^{-i \omega x_{0}}}{b+c^{2} \omega^{2}}\right\}=\frac{a}{2 \sqrt{b c}} e^{-\left|x-x_{0}\right| b^{1 / 2} / c},
$$

we get [28]

$$
\hat{p}_{0}\left(x, s \mid x_{0}\right)=\frac{s^{\alpha / 2-1}}{2 \sqrt{D}} e^{-\left|x-x_{0}\right| \sqrt{s^{\alpha} / D}} .
$$

The Laplace transform can also be inverted with the result [44]

$$
p_{0}\left(x, t \mid x_{0}\right)=\frac{1}{2 \sqrt{D t^{\alpha}}} M_{\alpha / 2}\left(\frac{\left|x-x_{0}\right|}{\sqrt{D t^{\alpha}}}\right),
$$

where $M_{\alpha / 2}(\cdot)$ is the Mainardi function defined by the power series $[42,45]$

$$
M_{\beta}(z)=\sum_{n=0}^{\infty} \frac{(-1)^{n} z^{n}}{n ! \Gamma(-\beta n+1-\beta)}, \quad 0<\beta<1 .
$$

Mainardi's function $M_{\beta}(z)$ is an entire function of $z$ for $0<\beta<1$ [42]. It is a special case of the Wright function $[45,46]$ which is closely related to the rather cumbersome Fox function, the latter frequently used in the anomalous diffusion literature [32].

The reset-free process is not stationary because as we can easily see from Eq. (44),

$$
p_{0}^{(\mathrm{st})}(x)=\lim _{t \rightarrow \infty} p_{0}\left(x, t \mid x_{0}\right)=0 .
$$

However, as we have seen in Sec. II, the addition of a resetting mechanism turns the process into an stationary one with a nonzero stationary density given by Eq. (16). For Poissonian resettings, cf. Eq. (14), the stationary density is given by Eq. (17), which for the anomalous diffusion process yields the tent-shape density [cf. Eq. (43)]

$$
p_{r}^{(\mathrm{st})}(x)=\frac{r^{\alpha / 2}}{2 \sqrt{D}} e^{-\left|x-x_{0}\right| \sqrt{r^{\alpha} / D}},
$$

an expression which has been very recently derived independently in Refs. [26,28].

\section{B. Survival probability}

The survival probability to some critical value $x_{c}$ of the underlying reset-free process, $S_{0}\left(t \mid x_{0}\right)$, is the solution to the following equation:

$$
\frac{\partial^{\alpha} S_{0}\left(t \mid x_{0}\right)}{\partial t^{\alpha}}=D \frac{\partial^{2} S_{0}\left(t \mid x_{0}\right)}{\partial x_{0}^{2}}
$$

$(0<\alpha<2)$, with the initial and boundary conditions

$$
S_{0}\left(t=0 \mid x_{0}\right)=1, \quad S_{0}\left(t \mid x_{c}\right)=0 .
$$

Similar to the case of the propagator discussed above, when $1<\alpha<2$ these conditions have to be supplemented with

$$
\left.\partial_{t} S_{0}\left(t \mid x_{0}\right)\right|_{t=0}=0
$$

which has implications in the behavior of $T_{r}\left(x_{0}\right)$, as we have seen [cf. Eqs. (31)-(33)].

In the Laplace space, and after using Eq. (41), this problem simply reads

$$
D \frac{\partial^{2} \hat{S}_{0}\left(s \mid x_{0}\right)}{\partial x_{0}^{2}}-s^{\alpha} \hat{S}_{0}\left(s \mid x_{0}\right)=-s^{\alpha-1}, \quad \hat{S}_{0}\left(s \mid x_{c}\right)=0 .
$$

As can be seen by direct substitution, the solution to this problem that is finite as $x_{0} \rightarrow \pm \infty$ is [28]

$$
\hat{S}_{0}\left(s \mid x_{0}\right)=\frac{1}{s}\left[1-e^{-\left|x_{0}-x_{c}\right| \sqrt{s^{\alpha} / D}}\right] .
$$

The Laplace inversion of this expression yields (see Ref. [44] for details)

$$
S_{0}\left(t \mid x_{0}\right)=1-\phi\left(-\alpha / 2,1,-\left|x_{0}-x_{c}\right| / \sqrt{D t^{\alpha}}\right),
$$

where $\phi(\rho, \beta, z)$ is the Wright function, which can be defined by the power series $[46,47]$

$$
\phi(\rho, \beta, z)=\sum_{n=0}^{\infty} \frac{z^{n}}{n ! \Gamma(n \rho+\beta)}
$$

$(\rho>-1, \beta>0)$.

The Laplace transform, $\hat{S}_{r}\left(s \mid x_{0}\right)$, of the survival probability to threshold $x_{c}$ for the complete process with resettings is 
given by Eq. (23), after substituting for $S_{0}\left(t \mid x_{0}\right)$ given in Eq. (51) and evaluating the Laplace transforms $\hat{h}\left(s \mid x_{0}\right)$ and $\hat{H}\left(s \mid x_{0}\right)$. The resulting expression is virtually impossible to handle for any resetting density $\psi_{r}(t)$. However, this can be easily done for Poissonian resettings. In such a case, substituting Eq. (50) into Eq. (34) yields

$$
\hat{S}_{r}\left(s \mid x_{0}\right)=\frac{1-e^{-(r+s)^{\alpha / 2}\left|x_{0}-x_{c}\right| / \sqrt{D}}}{s+r e^{-(r+s)^{\alpha / 2}\left|x_{0}-x_{c}\right| / \sqrt{D}}} .
$$

\section{Mean first-passage time}

We will first check that the MFPT for the reset-free process is infinite. In effect, expanding Eq. (50) for small values of $s$ we find

$$
\hat{S}_{0}\left(s \mid x_{0}\right)=\frac{\left|x_{0}-x_{c}\right|}{\sqrt{D}} s^{\alpha / 2-1}+O\left(s^{\alpha-1}\right) .
$$

Hence, for $0<\alpha<2$,

$$
T_{0}\left(x_{0}\right)=\lim _{s \rightarrow 0} \hat{S}_{0}\left(s \mid x_{0}\right)=\infty .
$$

For the combined process with resetting events governed by any switch density $\psi_{r}(t)$, the MFPT $T_{r}\left(x_{0}\right)$ is given by the general expression Eq. (26) after substituting $S_{0}\left(t \mid x_{0}\right)$ by Eq. (51). For Poissonian resettings we can write a simple and explicit expression for $T_{r}\left(x_{0}\right)$ since in this case taking the limit $s \rightarrow 0$ in Eq. (53) yields [28]

$$
T_{r}\left(x_{0}\right)=\frac{1}{r}\left[e^{\left|x_{0}-x_{c}\right| r^{\alpha / 2} / \sqrt{D}}-1\right] .
$$

From this expression we clearly see how $T_{r}\left(x_{0}\right)$ approaches $T_{0}\left(x_{0}\right)=\infty$ as $r \rightarrow 0$. Indeed,

$$
T_{r}\left(x_{0}\right)=\frac{\left|x_{0}-x_{c}\right|}{\sqrt{D}} r^{\alpha / 2-1}\left[1+O\left(r^{\alpha / 2}\right)\right],
$$

which diverges as $r \rightarrow 0$ for $0<\alpha<2$.

As we have shown above, the MFPT in this case is a nonmonotonous function of rate $r$ presenting a minimum for some rate located between 0 and $\infty .{ }^{8}$ For Poissonian resettings we can be more specific and obtain the minimum rate. In effect, in such a case the MFPT is given by Eq. (54) from which we can obtain an explicit expression for the derivative $\partial_{r} T_{r}\left(x_{0}\right)$ and the minimum rate $r_{m}$ will be the solution to the equation $\partial_{r} T_{r}\left(x_{0}\right)=0$. This elementary procedure leads to the transcendental equation ${ }^{9}$

$$
1-\frac{\alpha}{2} \xi=e^{-\xi}
$$

for the variable $\xi$ defined as

$$
\xi \equiv\left|x_{0}-x_{c}\right| r_{m}^{\alpha / 2} / \sqrt{D}
$$

Having obtained $\xi$ from the numerical solution of Eq. (55) for a given value of exponent $\alpha$, the minimum rate is thus given

\footnotetext{
${ }^{8} \mathrm{~A}$ similar approach to this issue has been very recently and independently derived in Ref. [28].

${ }^{9}$ In the particular case of ordinary diffusion, $\alpha=1$, Eq. (55) has been recently obtained in Ref. [15] with the result $\xi=1.594 \ldots$. .
}

by [28]

$$
r_{m}=\left(\frac{\xi \sqrt{D}}{\left|x_{0}-x_{c}\right|}\right)^{2 / \alpha}
$$

a result that we can replace back in Eq. (54) to obtain

$$
T_{\xi}\left(x_{0}\right)=\frac{\alpha \xi^{1-2 / \alpha}}{2-\alpha \xi} \cdot \frac{\left|x_{0}-x_{c}\right|^{2 / \alpha}}{D^{1 / \alpha}} .
$$

Note how the prefactor in this expression is a numeric quantity that only depends on $\alpha$.

\section{CONCLUDING REMARKS}

We have analyzed the effects of general resetting mechanisms on anomalous diffusion processes, specifically on the time-fractional Brownian motion. Although our primary purpose has been focusing on anomalous diffusion as underlying process, we have addressed the problem from a general point of view, assuming that both resettings and underlying processes are described in a general fashion with an arbitrary resetting density, $\psi_{r}(\tau)$, and an unspecified propagator, $p_{0}\left(x, t \mid x_{0}\right)$, for reset-free processes.

From this general analysis we have shown that, under rather general assumptions - basically the existence of a finite first moment for $\psi_{r}(\tau)$-resettings first stabilize the underlying process, in the sense that a nonstationary process becomes stationary under resettings. Second, resettings may greatly reduce the mean first-passage time to some critical value and presents a minimum value for some critical rate to be determined after the details of the whole process are known. This constitutes a complementary view of the universal character of resettings that has been recently brought forward in the literature [14,15,20-25].

We have finally performed a thorough study of the subject when the underlying process is a time fractional diffusion with exponent $\alpha \in(0,2)$, covering subdiffusion when $0<\alpha<1$ and superdiffusion when $1<\alpha<2$. The reset-free process possesses no stationary distribution and the mean first-passage time is infinite. We have shown that for Poissonian resettings the stationary distribution has the form of a tent-shape density (i.e., Laplace distribution) given in Eq. (46). We have finally obtained explicit expressions for the survival probability-in Laplace space [Eq. (53)] — and the mean first-passage time [Eq. (54)]. We have shown that this is a nonmonotonous function of the resetting rate $r$ and have obtained the minimum rate.

\section{ACKNOWLEDGMENTS}

The authors acknowledge partial support from the Spanish Agencia Estatal de Investigación and the Fondo Europeo de Desarrollo Regional (AEI/FEDER, UE) under Contract No. FIS2016-78904-C3-2-P, and from the Catalan Agència de Gestió d'Ajuts Universitaris i de Recerca (AGAUR), Contract No. 2017SGR1064. 
[1] A. Badrisarayanan, T. B. Le, and M. T. Laub, J. Cell Biol. 210, 385 (2015).

[2] S. Reuveni, M. Urbalch, and J. Klafter, Proc. Natl. Acad. Sci. USA 111, 4391 (2014).

[3] E. Roldán, A. Lisica, D. Sánchez-Taltavull, and S. W. Grill, Phys. Rev. E 93, 062411 (2016).

[4] V. Méndez, D. Campos, and F. Bartumeus, Stochastic Foundations in Movement Ecology: Anomalous Diffusion, Front Propagation and Random Searches (Springer-Verlag, Berlin, 2013).

[5] A. Reynolds, Phys. Life Rev. 14, 59 (2015).

[6] M. Luby, A. Sinclair, and D. Zuckerman, Inf. Proc. Lett. 47, 173 (1993).

[7] A. Montanari and R. Zecchina, Phys. Rev. Lett. 88, 178701 (2002).

[8] M. Tong, C. Faloutsos, and J.-Y. Pau in Proceedings of 6th International Conference on Data Mining (IEEE Computer Society, Hong Kong, 2006).

[9] M. R. Evans and S. N. Majumdar, Phys. Rev. Lett. 106, 160601 (2011).

[10] M. R. Evans and S. N. Majumdar, J. Phys. A 47, 285001 (2014).

[11] A. Pal, A. Kundu, and M. R. Evans, J. Phys. A 49, 225001 (2016).

[12] M. Montero and J. Villarroel, Phys. Rev. E 87, 012116 (2013).

[13] Ł. Kuśmierz and E. Gudowska-Nowak, Phys. Rev. E 92, 052127 (2015).

[14] S. Belan, Phys. Rev. Lett. 120, 080601 (2018).

[15] S. Reuveni, Phys. Rev. Lett. 116, 170601 (2016).

[16] T. S. Biró and J. Néda, Physica A 499, 335 (2018).

[17] A. Pal, Phys. Rev. E 91, 012113 (2015).

[18] C. Christou and A. Schadschneider, J. Phys. A: Math. Theor. 48, 285003 (2015).

[19] J. Masoliver, Phys. Rev. E 99, 012121 (2019).

[20] T. Rotbart, S. Reuveni, and M. Urbakh, Phys. Rev. E 92, 060101(R) (2015).

[21] S. Eule and J. J. Metzger, New J. Phys. 18, 033006 (2016).

[22] A. Nagar and S. Gupta, Phys. Rev. E 93, 060102(R) (2016).

[23] A. Pal and S. Reuveni, Phys. Rev. Lett. 118, 030603 (2017).
[24] A. Chechkin and I. M. Sokolov, Phys. Rev. Lett. 121, 050601 (2018).

[25] J. Villarroel and M. Montero, J. Stat. Mech. (2018) 123204.

[26] A. Masó-Puigdellosas, D. Campos, and V. Méndez, Phys. Rev. E 99, 012141 (2019).

[27] S. N. Majumdar and G. Oshanin, J. Phys. A 51, 435001 (2018).

[28] Ł. Kuśmierz and E. Gudowska-Nowak, Phys. Rev. E 99, 052116 (2019).

[29] D. ben-Avraham and S. Havlin, Diffusion and Reactions in Fractals and Disordered Systems (Cambridge University Press, Cambridge, 2000).

[30] R. Balescu, Aspects of Anomalous Transport in Plasmas (Taylor \& Francis, London, 2005).

[31] J. P. Bouchaud and A. Georges, Phys. Rep. 195, 127 (1990).

[32] R. Metzler and J. Klafter, Phys. Rep. 339, 1 (2000).

[33] J. Klafter and I. M. Sokolov, Phys. World 18, 29 (2005).

[34] R. Balescu, Chaos Solitons Fractals 34, 62 (2007).

[35] I. I. Eliazar and M. F. Shlesinger, Phys. Rep. 527, 101 (2013).

[36] B. J. West, Rev. Mod. Phys. 86, 1169 (2014).

[37] Y. Meroz and I. Sokolov, Phys. Rep. 573, 1 (2015).

[38] E. W. Montroll and G. H. Weiss, J. Math. Phys. 6, 167 (1965).

[39] J. Masoliver, Phys. Rev. E 93, 052107 (2016).

[40] A. S. Bodrova, A. V. Chechkin, and I. M. Sokolov, Phys. Rev. E 100, 012119 (2019); 100, 012120 (2019).

[41] K. Jacobs, Stochastic Processes for Physicists (Cambridge University Press, Cambridge, 2010).

[42] F. Mainardi, Appl. Math. Lett. 9, 23 (1996).

[43] I. Podlubny, Fractional Differential Equations (Academic Press, San Diego, 1999).

[44] J. Masoliver, Random Processes, First-Passage and Escape (World Scientific, Singapore, 2018).

[45] F. Mainardi and G. Pagnini, J. Comput. Appl. Math. 207, 245 (2007).

[46] A. Erdelyi et al., Higher Transcendental Functions, Vol. 3 (McGraw-Hill, New York, 1953).

[47] R. Gorenflo, Y. Luchko, and F. Mainardi, Fract. Calc. Appl. Anal. 2, 383 (1999). 